\title{
Implementation of Multi-Body Dynamics Simulation for the Conveyor Chain Drive System
}

\author{
Chakrit Suvanjumrat, Wasan Suwannahong and Sommart Thongkom \\ ${ }^{1}$ Department of Mechanical Engineering, Faculty of Engineering, Mahidol University, Salaya, Nakorn Pathom, 73170, Thailand \\ ${ }^{2}$ Laboratory of Computer Mechanics for Design (LCMD), Department of Mechanical Engineering, Faculty of Engineering, Mahidol University, \\ Salaya, Nakorn Pathom, 73170, Thailand
}

\begin{abstract}
The conveyor chain drive system which included four sprockets, four flat bars (chain guides) and one conveyor chain had been assembled into commercial software, MSC.ADAMS. The conveyor chain was connected with rollers for running on the chain guides. The multi-body dynamics (MBD) simulation was implemented to study the large displacement of chain components which happened during rotations of the symmetric model of the conveyor chain drive system. The physical experiment had obtained by construction of the conveyor chain drive system regarding the MBD model. The speeds of chain drive were adjusted by a gear motor with a converter. The trajectory of conveyor chain links which respectively meshed and ran on sprockets and chain guides was recorded by a high speed camera. Furthermore, impact-contact forces by a collision of components in the conveyor chain drive system during rotation were analysis. The comparison between MBD simulation and physical experiment of the conveyor chain drive system was performed for validation of simulation models. The MBD simulation results were in good agreement with the experimental data which obtained an average error of $3.95 \%$.
\end{abstract}

\section{Introduction}

The conveyor chain has used in a long distance of producing lines to transport products or parts which have a heavy load. This conveyor chain has many components which build up to move and hold products. The contactimpact between conveyor chain and other components in producing line always happen which induces noise and fatigue damage of the conveyor chain system. The conveyor chain in producing line is an enormous system, therefore, complex to analyze with simulation method such as finite element analysis (FEA). The MBD simulation is widely used to model the different body motion for each component of the large system [1], [2]. The constraints which relate with bodies are used to limit motions of the system. The contact-impact constraint is a major condition to detect the precise of impact and to achieve the contact-impact force by MBD simulation [3]. The dynamic characteristics of movable components in systems can be investigated. The dynamic path, force and moment are the whole results which achieve by the MBD simulation to improve rigid components of the dynamic system [4], [5].

In this research, MBD simulation for the conveyor chain system had performed by using MSC.ADAMS software. The trajectory of MBD simulation was validated with the physical experiment. The impactcontact force which happened between contact components was studied. The simulation of the conveyor chain drive system will be used to design and analyze the larger conveyor chain drive system of the rubber glove production line in the further works.

\section{Theory of multi-body dynamics}

Multi-body dynamics system is a whole system composed of multiple bodies which are combined by the series of geometry constraints to calculate an expected motion. The bodies in multi-body dynamics system concern rigid bodies to form equation of motion by the Lagrangian equation [6]. The dynamic equation of each body of the system is given by:

$$
\frac{d}{d t}\left(\frac{\partial L}{\partial \dot{x}_{k}}\right)-\frac{\partial L}{\partial x_{k}}=F_{k} ; k=1,2,3, \cdots, n
$$

where

$$
L=T-U
$$

where $x_{k}$ is the generalized coordinate of the $k$-th body, $F_{k}$ is the generalized force on $k$-th body, $n$ is number of bodies, $L$ is the Lagrangian function, $T$ is kinetic energy, $U$ is potential energy.

The constraint equation relates with bodies is given to assemble bodies by:

$$
C_{i}(x, t)=0 ; i=1,2,3, \cdots, m
$$

where $m$ is the number of constraints. 
The dynamic equation of system is expressed in the matrix form by:

$$
\frac{d}{d t}\left(\frac{\partial T}{\partial x_{k}}\right)+K x_{k}=F_{k}
$$

where $\boldsymbol{K}$ is the rigidity matrix. $\boldsymbol{F}_{k}$ is the force vector.

The bodies are assembled to a system through constraints. The differential equation of the system is generated by the Lagrange multiplier method which written by matrix form as:

$$
\boldsymbol{M} \ddot{x}_{k}+\boldsymbol{K} x_{k}+\boldsymbol{J}_{C}^{T} \lambda=\boldsymbol{F}_{k}
$$

where $\boldsymbol{M}$ is the mass matrix. $\boldsymbol{I}_{C}$ is the Jacobian matrix of constraints. This dynamic governing equation of the system will solve by the numerical technique through MSC.ADAMS in this research.

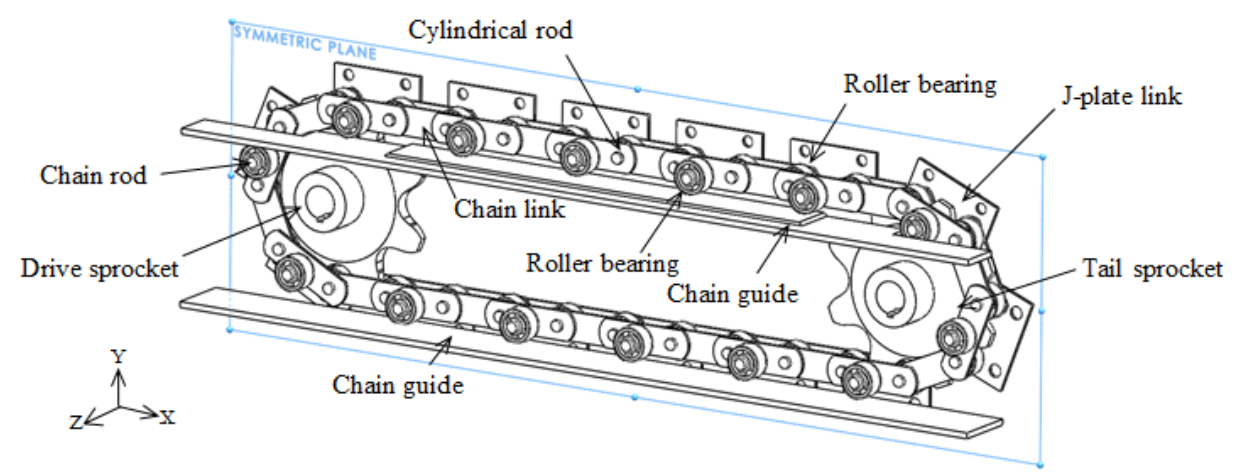

Figure 1. The MBD model of the conveyor chain drive system.

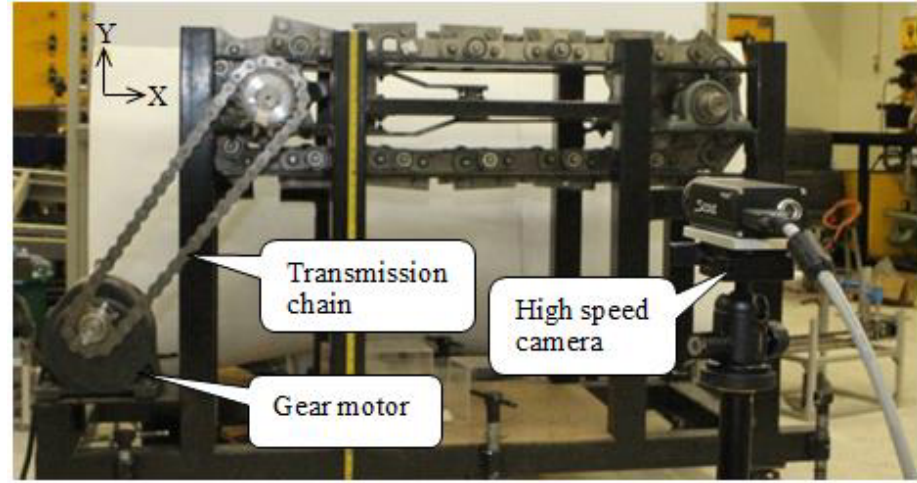

Figure 2. Apparatus of the conveyor chain drive system for validation of the MBD simulation.
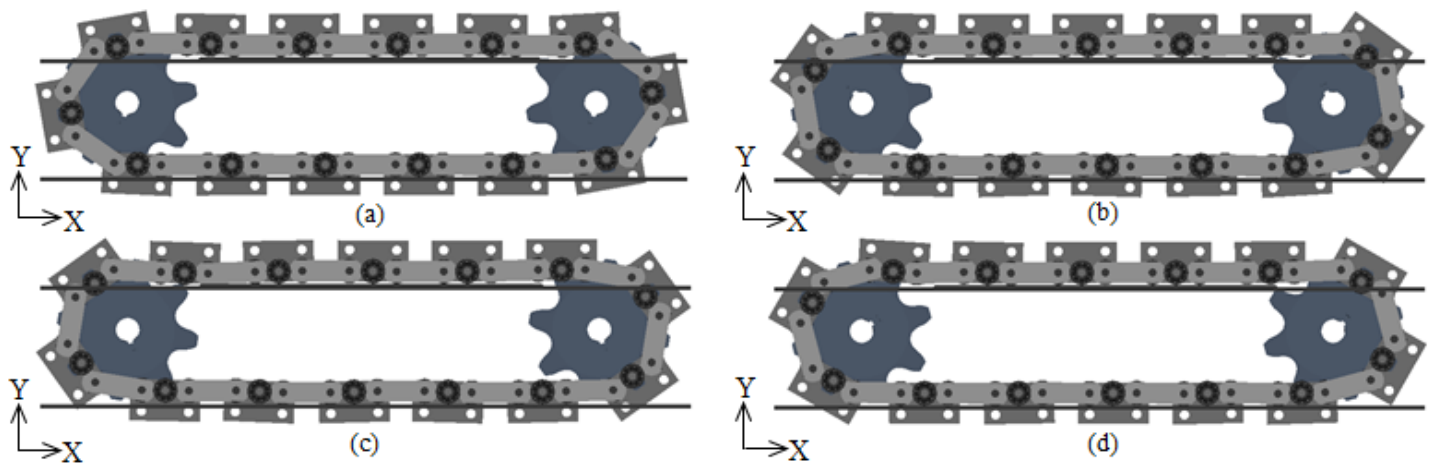

Figure 3. The sequent image at: (a) time $=0.06 \mathrm{sec}$, (b) time $=0.76 \mathrm{sec}$, (c) time $=1.78 \mathrm{sec}$ and (d) time $=3.04 \mathrm{sec}$ of the conveyor chain drive system rotating with the rotational speed of $3.40 \mathrm{rad} / \mathrm{s}$.

\section{Multi-Body dynamics simulation}

The computer aided design (CAD) model of the conveyor chain drive system was created by SolidWorks software which comprises of sprockets, conveyor chain and chain guides. This CAD model was imported into MSC.ADAMS to create the MBD model for the first step of the modeling process. Fig. 1 shows the MBD model of the conveyor chain drive system. The symmetric plane was used to model the conveyor chain drive system to reduce the processing time. The conveyor chain components were assembled including roller bearings, cylindrical rods, chain links and J-plate links. Total components of the symmetric conveyor chain model which wrap around two sprockets were 126 pieces. The conveyor chain which was modeled for the MBD simulation composed of 28 links. The roller bearings which wore chain rods would roll on the chain guide. The contact-impact constraint between components such as roller bearings and chain guides, cylindrical rods and $\mathrm{J}$ plates, roller bearings and sprocket tooth and chain links and roller bearings were carefully built to prevent the software to automatically detect and remove any 
redundant constraints. The input of the MBD model was the rotational speed of the driving sprocket. The 8-teeth sprocket in front of the conveyor chain drive system drove the chain in the clockwise direction with the angular velocity of $1.46,2.33$ and $3.40 \mathrm{rad} / \mathrm{s}$. The component of the conveyor chain drive system had been used material density to specify weight by multiplying with the component volume and the gravitational acceleration. The personal computer with core-i5 CPU and RAM memory of $4 \mathrm{~GB}$ was used to perform the MBD simulation. The roller bearing which meshed sprocket tooth was calculated the trajectory to compare with the experimental data. The trajectory of roller bearing which rolled on chain guides also was calculated and corrected to compare with the physical experiment. The contact-impact force which immediately happened when bearings contacted and impacted with chain guides and sprocket tooth was gathered for calculation to analyze the dynamic appearance of the conveyor chain drive system.

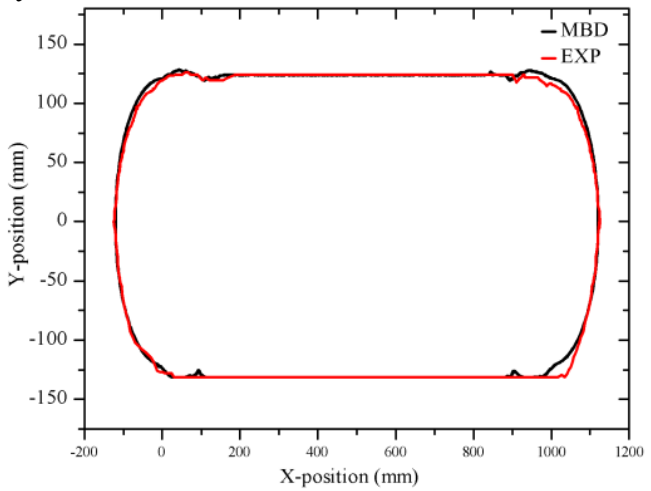

(a)

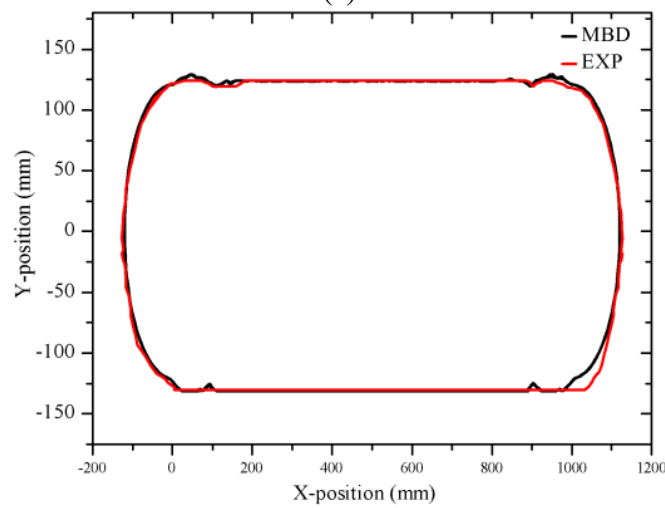

(b)

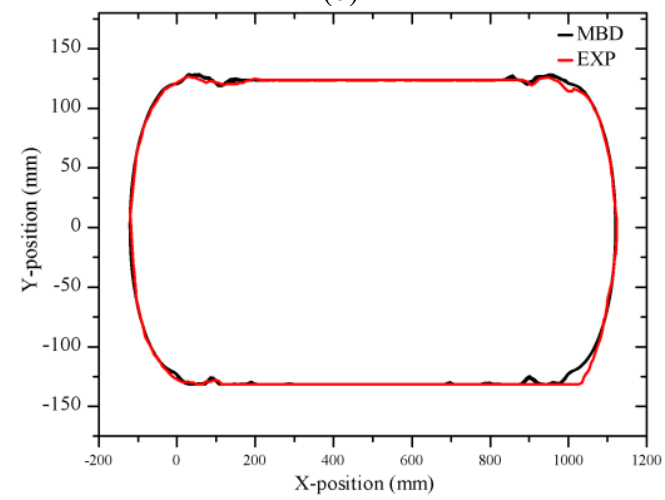

(c)

Figure 4. The path of the roller bearing meshing sprockets at the angular velocity of: (a) 1.46 , (b) 2.33 and (c) $3.40 \mathrm{rad} / \mathrm{s}$.

\section{Experiment}

The conveyor chain drive system is constructed and shown in Fig. 2. The eight-leg frame which built by the $4 " \times 4$ " square steel tube was supported fully components of the conveyor chain drive system. The driving sprocket was driven by a gear motor through a small chain. The motor speed was controlled by a motor converter which installed in a control box. The motor speed had been adjusted to rotate drive sprockets according to the angular velocity of the MDB simulation of the conveyor chain drive system for investigating the variable dynamic behavior of the conveyor chain. The high speed camera (BASLER Scout scA640-70fm) was set up to record the conveyor chain driving by using a frame rate and image resolution of $71 \mathrm{fps}$ and $659 \times 494$ pixels, respectively.

\section{Results and discussion}

The conveyor chain drive system was controlled to rotate with the different rotational speed. Fig. 3 shows sequent images of the conveyor chain drive system rotating with the rotational speed of $1.23 \mathrm{rad} / \mathrm{s}$. The path of a center position of roller bearings which are meshed sprockets has been plotted to compare with experimental data as shown in Fig. 4. The X-Y path of roller bearings which run on chain guides is plotted by comparison graphs between MBD simulation and physical experiment as in Fig. 5. The oscillation of roller bearing was increased following the angular velocities. The comparison shows the MBD simulation result was in a good agreement with the experimental data. The path of bearings is used to calculate distance from a center point of bearings to a center point of the driving sprocket by:

$$
D_{c}=\sqrt{X^{2}+Y^{2}}
$$

where $X$ is the distance of bearings from the center point of the driving sprocket in $\mathrm{x}$-direction, $Y$ is the distance of bearings from the center point of the driving sprocket in y-direction. The error of the MBD simulation was used the comparison of the distance of bearing movement between the MBD model and the physical experiment. The roller bearing of MBD model which meshed sprockets obtained an average error of $4.73,3.47$ and $3.11 \%$ while roller bearing which ran on chain guides obtained an average error of $2.50,5.70$ and $4.22 \%$ under the rotational speed of $1.46,2.33$ and $3.40 \mathrm{rad} / \mathrm{s}$, respectively.

The maximum contact-impact force occurred on the bearings which was connected to chain links and meshed with the sprocket tooth. The magnitude of contact-impact force which varies along the rotational time of the conveyor chain drive system can express by the graph as shown in Fig. 6. The magnitude of contact-impact force was increased while the time period was reduced when increased angular velocities of drive sprockets. The contact-impact forces on chain guides were very less than on sprockets. Fig. 7 shows contact-impact forces of bearings which roll on the chain guides. 


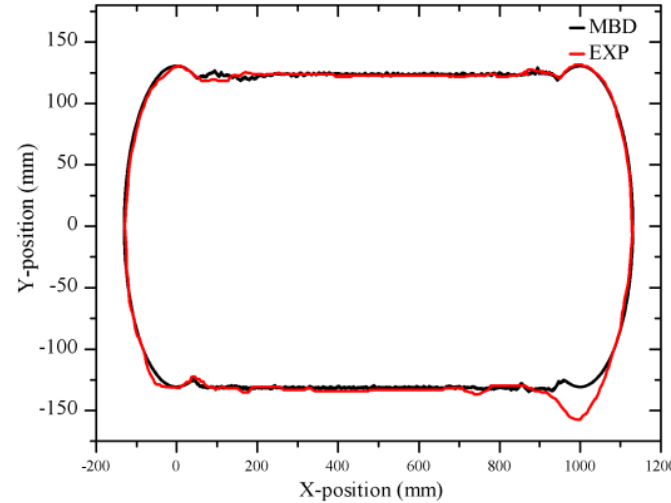

(a)

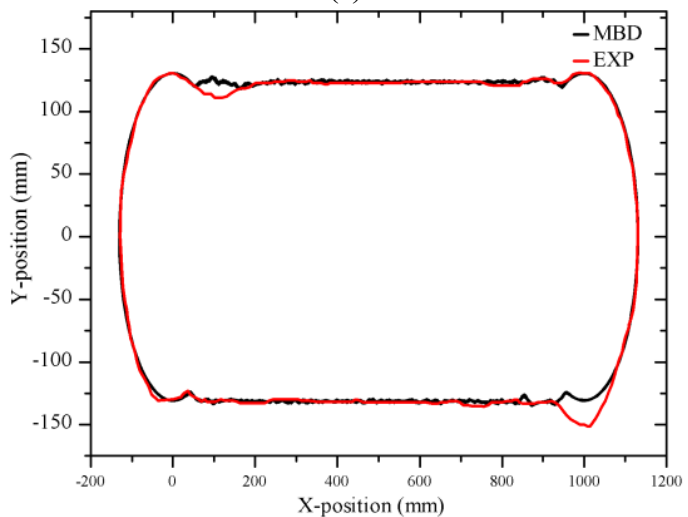

(b)

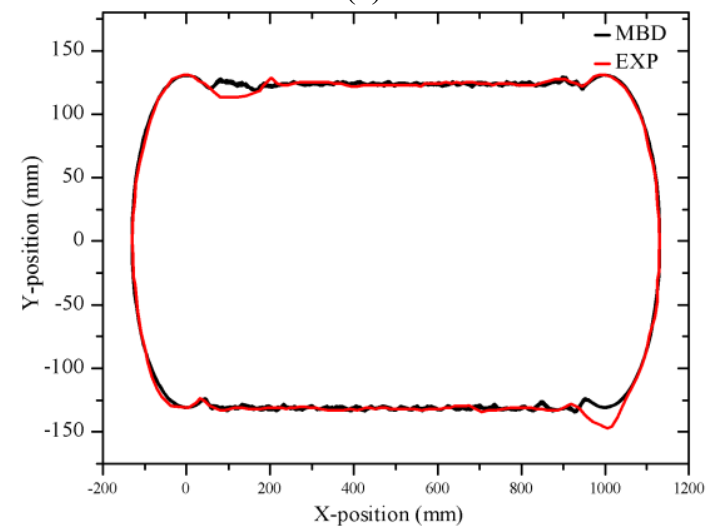

(c)

Figure 5. The path of the roller bearing running on chain guides at the angular velocity of: (a) 1.46 , (b) 2.33 and (c) $3.40 \mathrm{rad} / \mathrm{s}$.

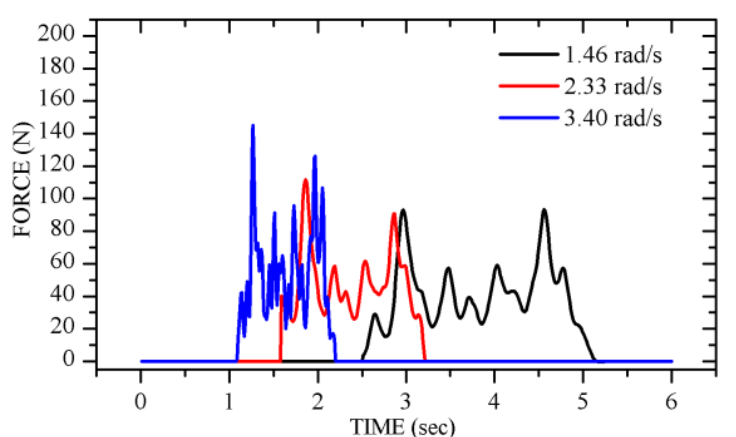

Figure 6. The contact-impact force of the roller bearings during meshing with the sprocket tooth.

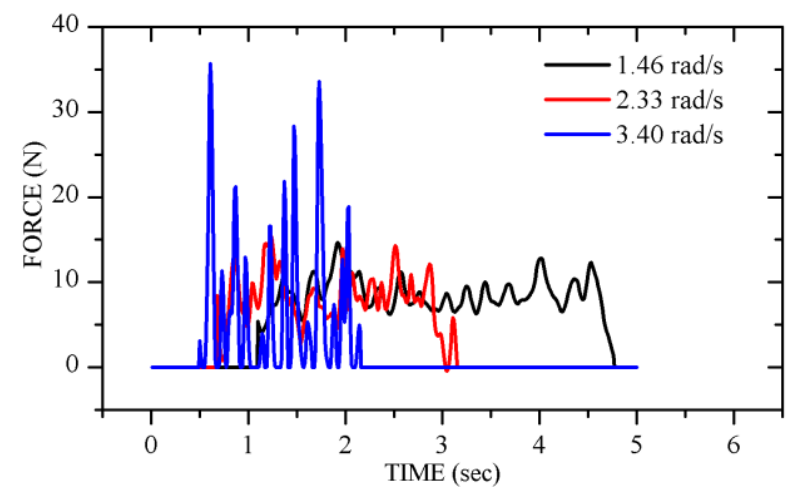

Figure 7. The contact-impact force of the roller bearings during running on the chain guides

\section{Conclusions}

The MBD simulation of the conveyor chain drive system was performed by using the commercial software, MSC.ADAMS. The physical experiment of the conveyor chain drive system had been constructed to validate the MBD simulation results. The path of the bearings which was the component of the system had been used to validate by comparison between simulation results and experimental data. The MBD simulation results were in a good agreement with experimental data and obtain an average error less than $3.95 \%$. The simulation results shown the oscillation of components increased by increasing of the angular velocities of the drive sprockets. The MBD simulation not only calculated path of components but also the contact-impact force between components could also achieve. The large contact-impact force happened on sprockets of the conveyor chain drive system. The MBD simulation would be useful and advance to design and calculate the strength of the conveyor chain drive system for the producing line in a further work.

\section{References}

1. C.J. Park, G. Gschwendtner, J. Mech. Sci. Technol. 29, 7 (2015)

2. Y. Li, A.M. Castro, T. Sinokrot, W. Prescott, P.M. Carrica, Renew. Energ. 76 (2015)

3. P. Flores, J. Ambrosio, Multibody Syst. Dyn. 24 (2010)

4. M. Schwaze, C. Hurschler, F. Seehaus, S. Oehler, B. Welke, J. Biomech. 46 (2013)

5. E. Abele, J. Bauer, T. Hemker, R. Laurischkat, H. Meier, S. Reese, O. von Stryk,CIRP-JMST. 4 (2011)

6. F.E. Udwad, A.D. Schutte, Acta. Mech. 213 (2010) 